\title{
Archivos y centros de documentación en la prensa local de la Comunidad de Madrid
}

\author{
David RodRÍGUEZ MATEOS \\ david.rodriguez@uc3m.es \\ Tony HERNÁNDEZ PÉREZ \\ antonio.hernandez@uc3m.es \\ Eva MÉNDEZ RoDRÍGUEZ \\ emendez@bib.uc3m.es \\ Universidad Carlos III de Madrid
}

Recibido: $15 / 06 / 2012$

Aceptado: 17/07/2012

\section{RESUMEN}

El texto presenta una introducción sobre la situación actual de los medios de comunicación locales en la Comunidad de Madrid y los resultados de un estudio sobre las prácticas de uso de los archivos y centros de documentación que se llevan a cabo en 12 medios periodísticos locales radicados en la Comunidad de Madrid. El trabajo se llevó a cabo mediante visitas y entrevistas a responsables periodísticos de dichos medios. Entre los resultados obtenidos destaca el uso masivo de tecnologías informáticas y la falta de conciencia de los periodistas locales sobre el valor histórico y las posibilidades de un tratamiento normalizado de la documentación.

Palabras clave: Periodismo local, documentación periodística, Comunidad de Madrid, Internet, archivo, tecnologías de la información

\section{Local media archives in the Region of Madrid}

\begin{abstract}
This article offers an introduction about the current situation of local press in the Madrid area as well as the results of a study about the use and practice in their archives and documentation centers. The study was made in 12 of the main Madrid local press groups by means of interviews and visits to those media. Among the results obtained it's worth to highlight the massive use of information technologies and the lack of awareness of the media about the historic value and the possibilities of a standardized treatment of the documents.
\end{abstract}

Keywords: Local press, Madrid, Internet, information technologies, archives, documentation 


\section{INTRODUCCIÓN}

De acuerdo a los datos del Estudio General de Medios (AIMC - EGM, 2011) entre octubre de 2010 y mayo de 2011 la audiencia de los diarios en la población española descendió un 3\% respecto al período analizado de octubre 2009 a junio de 2010. En ese período El País ha perdido el 2' $5 \%$ de audiencia, El Mundo ha perdido un 9'3\% de su audiencia o $\mathrm{ABC}$, que ha perdido un $71 \%$. Y si las caídas de audiencia son generalizadas entre los diarios de pago no lo son menos entre los diarios gratuitos y los diarios locales (20 Minutos, -7'5\%; Qué, $-9^{\prime} 6 \%$; o ADN, $-15^{\prime} 6 \%$ ) los que más lectores nuevos aportaron durante la etapa de crecimiento de audiencia de la prensa escrita entre 2001 y 2008, una etapa caracterizada por la aparición de nuevos medios de prensa gratuita con orientación hacia lo local, casi de distrito, y dirigido en muchos casos a una población inmigrante.

El periodismo local o de proximidad está viviendo un momento de cambios: por la incertidumbre introducida por la crisis económica general. La disminución de la publicidad, tanto institucional como comercial, afecta gravemente al periodismo local. De acuerdo a un informe de Zenith Vigía (PRNoticias, 2011), una empresa especializada en el sector publicitario, la inversión en publicidad en este año 2011 podría crecer, de acuerdo a las estimaciones más optimistas, un 0 '2\% en la televisión generalista, un $3 \%$ en la temática. Y "se espera además descensos ligeros $(-0,3 \%)$ en el caso de la Radio y algo mayor para los demás medios. Exterior (-1,8\%), Revistas $(-4,9 \%)$; Cine (-5,0\%); Suplementos (-5,5\%); Televisión Local (-7,2\%); Diarios: de Pago ($7,8 \%)$ y Gratuitos $(-10,1 \%)$ y Medios para Inmigrantes $(-8,5 \%)$ ".

A esta crisis económica generalizada, el sector de la prensa gratuita y de la prensa local suma las "profundas transformaciones marcadas por los procesos de diversificación y convergencia tecnológica; de concentración empresarial; por la evolución de los contenidos hacia formatos que alcancen audiencias cada vez más segmentadas y específicas; por los problemas de los puntos de distribución convencionales para la prensa de pago y por los nuevos sistemas de distribución para los medios gratuitos" (Peinado, 2010). A ello se suma el aumento del número de medios locales, con el surgimiento de nuevas cabeceras accesibles únicamente a través de Internet (López García, 2008).

También les afecta una crisis específica común a todos los medios de comunicación y que debe explicarse no tanto en términos económicos como sociológicos: son los cambios en los estilos de vida que están afectando al consumo de información, especialmente en el acceso a la información a través de dispositivos móviles y lo que eso supone en cuanto a los cambios no sólo en cómo se accede a la información sino también en cuanto a cómo se consume.

Lo que ha diferenciado al periodismo local o periodismo de proximidad (Izquierdo Labella, 2010) ha sido que tanto el sujeto (los periodistas y las fuentes que informan), como el objeto (los temas sobre los que se informa), como el público (las personas a las que se informa) comparten un mismo espacio geográfico, cercano y acotado. In- 
ternet facilita ahora que esos espacios físicos se difuminen. Ahora en un barrio de Madrid un periódico puede informar fácilmente de las ligas de fútbol rumana o latinoamericana porque el público al que se informa procede de esos países pero tiene un nuevo competidor: los mismos periódicos y medios que utiliza como fuente para informar y que también están accesibles por Internet directamente para los usuarios.

Pero también una audiencia más fiel y más diversa: el lector local, y el visitante que viaja o se desplaza de forma más o menos temporal, puede ahora mantenerse informado acerca de aquello que, aunque no esté físicamente en un lugar, sigue perteneciendo a su momento vital y a su entorno más cercano. Y ya no necesita la cercanía física al lugar de distribución del medio, ni para leer la prensa, ni para escuchar la radio ni para ver la televisión. De hecho, ésta ha sido una de las tendencias encontradas en nuestro estudio: los medios de comunicación locales son, cada vez más, multimedios, con prensa impresa pero también con canales de televisión por Internet, emisoras de radio por la red, y versiones web de lo que publican en impreso.

La digitalización y la irrupción de Internet está permitiendo a los medios explotar sus fondos de archivo, generalmente a través de la reutilización de información, lo que permite aumentar y alimentar los flujos de información que ofrece a sus audiencias a un coste prácticamente inexistente.

El objetivo fundamental de este trabajo es presentar una nueva radiografía sobre los archivos y servicios de documentación en los medios específicamente locales de la comunidad madrileña, es decir, sobre aquellos que acotan su ámbito de influencia a la región, en todo o en parte, y analizar la existencia y el uso que han estado haciendo de sus archivos y/o servicios de documentación. El trabajo presenta un estudio general que abarca el mayor número de aspectos posibles, entre los que puede destacarse: la presencia de los centros de documentación en los medios locales; el uso de los mismos para la creación de información local; la infraestructura material empleada para ello; la influencia de las tecnologías de la información sobre la actividad documental y sobre la consulta de información; y por último, las perspectivas ante el futuro, especialmente, en momentos de crisis económica que está afectando seriamente a la supervivencia de muchos de estos medios.

La documentación periodística ha contado con notable literatura al respecto, entre las que destacan algunas relativas a la relación entre documentalistas y periodistas (Paul, 2009). Varias de las publicaciones al respecto, sobre medios concretos, están enfocadas a medios autonómicos, y especialmente, a televisiones autonómicas, como la asturiana (Estrada Nora-González y otros, 2009), la valenciana (Alfonso-Noguerón, 2009) la catalana (Conesa, 2006) o la gallega (López Elvira, 2005). También se cuentan análisis concretos sobre ejemplos de periódicos locales (Ros-Martín y otros, 2009).

Más específicamente, entre las referencias relativas a la documentación en medios locales de comunidades autónomas, contamos como referencias sobre los medios locales en el País Vasco (Agirreazaldegi Berriozábal y otros, 2009) y, más lejanas en el tiempo, las televisiones locales de Cataluña (Franganillo, 1999), los medios de 
Andalucía (Aquesolo, 1996), algunos medios de comunicación radicados en Madrid (Razquín, 1993) o los diarios locales de Barcelona (Fuentes i Pujol, 1992), por citar algunos casos.

\section{METODOLOGÍA}

La investigación se enmarca dentro de un proyecto conjunto con las universidades de A Coruña y la Autónoma de Barcelona para estudiar la situación de los archivos y servicios de documentación en las comunidades de Madrid, Cataluña y Galicia. Para la observación, se eligió una muestra de quince medios de comunicación por cada comunidad autónoma y la aplicación de un cuestionario común a los tres territorios. La recogida de la información se realizó fundamentalmente mediante entrevistas personales (aunque en algún caso, se recurrió a la entrevista telefónica) a diversos responsables periodísticos de cada medio y, cuando fue posible, también a personas que trabajaran en centros de documentación de los mismos.

En la Comunidad de Madrid se dan algunas pautas que son exclusivas respecto a otras comunidades en cuanto al empleo de la documentación en los medios locales, probablemente relacionado con la competencia y con la existencia de los grandes grupos de comunicación en la capital de España y con los hábitos de los lectores madrileños. En Madrid, "la información local y de distrito se dinamizó a partir del año 2002 con la edición de nuevos títulos de prensa gratuita local como El Nuevo Norte de Madrid y durante los siguientes cuatro años El Distrito y Gente van a segmentar geográficamente la capital con diferentes cabeceras de información local y por distritos, y coinciden con el nacimiento de los diarios gratuitos generalistas nacionales ADN y Qué que se sumaron a los ya existentes 20 Minutos y Metro. Pero esta expansión de la prensa de información general y local se frena en 2006, ante el principio de saturación de los diarios gratuitos generalistas (que terminó con el cierre de Metro) y una crisis que se avecinaba y que dará sus primeros zarpazos a la prensa gratuita local". (Peinado, 2010)

La investigación del proyecto, en el conjunto de las tres comunidades autónomas analizadas, se centró principalmente en una serie de medios que pudieran ser considerados como "locales": es decir, que tuvieran su sede central en alguna localidad dentro del radio de acción de la comunidad autónoma analizada (pudiendo tener delegaciones en otras partes de la misma, en algún caso); que no dependieran de otra sede central que estuviera fuera de la comunidad autónoma analizada; y que la mayor parte de su contenido emitido o publicado fuera de ámbito local.

La realidad del periodismo local en las tres comunidades autónomas presentaba algunas diferencias: respecto a la Comunidad de Madrid (que tiene mayor presencia de prensa gratuita y local, unas 140 cabeceras en 2011), existía una mayor presencia de radio y televisión local en Cataluña (Corominas, 2009), y, en menor medida, en 
Galicia, donde algunas fuentes hablaban de medios locales audiovisuales en desaparición (Pérez Feijoo, 2008).

Este mayor número de medios, entre otras razones, se debía a: la existencia de más de un idioma de referencia en aquellas, lo cual supone que la promoción de sus respectivas lenguas territoriales sea un factor añadido para el mantenimiento de la presencia de estos medios, mediante apoyos tanto públicos (Fundació Espai Català de Cultura y Comunicació, 2009) como privados; a una menor presión de medios nacionales en aquellas dos comunidades, que aumenta el mercado natural para estos medios; y a una presencia en ambas de diarios regionales o provinciales que evitan una segmentación en prensa tan grande como ocurre en Madrid. De hecho se estima que la tirada conjunta de los diarios gratuitos de información general en Madrid representa el 35,7\% del total de la difusión controlada por PGD/Introl, la principal institución que controla la difusión de la prensa tanto impresa como digital en toda España, y eso que sólo 71 cabeceras (el 50’7\%) son controladas por PGD/Introl, a través de su encuesta OJD, en el caso de la prensa gratuita (Peinado, 2010).

Sin embargo, la intención de obtener una representación equilibrada de los medios analizados en el estudio llevó a que se decidiera, en cada una de las tres comunidades, elegir una muestra similar de medios a analizar: un total de 15 medios por comunidad. En el caso de la Comunidad de Madrid, debido al predominio de los medios de comunicación de prensa y, en menor medida, de radio, apenas quedan canales de televisión locales que cumplan los requisitos planteados. Por otra parte, surgió una cierta dificultad para obtener datos concretos por diversas razones en algunos medios, entre la elección de cada medio al comienzo de la investigación y su contacto con el mismo: durante ese periodo, en algún caso, se había producido su cierre. Finalmente, con la intención de no desequilibrar la muestra final, ésta se redujo a 12 medios:

- uno de ellos es un periódico local, El Telégrafo (publicado en Collado Villalba).

- otros seis son medios que pertenecen a grupos de prensa con varias publicaciones locales en la Comunidad de Madrid: cuatro, en diferentes barrios de la ciudad de Madrid: Gaceta Local, Crónica de Madrid, Santa Eugenia y El Distrito; otro, La Noticia del Henares, en Alcalá de Henares; y uno más, Al Día Noticias, en Getafe.

- dos son grupos multimedia que incluían prensa y radio: El Iceberg (Griñón) y Radio Pueblo Nuevo (Madrid).

- dos son emisoras de radio (ambas, públicas y municipales): Onda Aranjuez (Aranjuez) y Radio Villalba (Collado Villalba).

- un grupo abarca radio y televisión: el ente público autonómico, que incluye Telemadrid (televisión) y Onda Madrid (radio). Este grupo se eligió debido a que su ámbito, a pesar de exceder el área meramente local, forma parte también de lo que puede considerarse como medios de proximidad, especialmente, porque cubre información local con mucha mayor frecuencia que los me- 
dios nacionales, al igual que ocurre en otras comunidades autónomas, como Cataluña (Domingo y otros, 2009), y porque la oferta televisiva específicamente local en la Comunidad de Madrid, fruto de la revisión previa de los medios existentes en esta región, es bastante escasa.

Una vez delimitada la muestra, se procedió a la elaboración de dos cuestionarios de preguntas: uno, dirigido a los responsables periodísticos de los medios respecto a sus fondos documentales, su utilización, su grado de importancia, la consideración de los profesionales encargados de su manejo en el medio correspondiente, la influencia de las tecnologías de la información en su propia forma de manejo de la información y en la ayuda de los documentalistas a este respecto, así como algunas cuestiones sobre las perspectivas de futuro. Y otro cuestionario similar, adaptado a sus tareas, más dirigido a los responsables o a los trabajadores de los propios centros de documentación cuando los hubiese.

\section{EL PERIODISMO LOCAL: UN ENTORNO EN TRANSFORMACIÓN}

El periodismo local no escapa a los fuertes aires de cambio que afectan al periodismo en general y actualmente se encuentra inmerso en los últimos años en un claro período de transformación (López Lita y otros, 2007). Esta transformación se debe a la suma de varios factores: en primer lugar, por una crisis económica general cuya intensidad está afectando a la existencia de muchas empresas periodísticas, que están viendo como su principal fuente de ingresos, la publicidad, cae de forma drástica, especialmente en el sector institucional, junto con la publicidad del pequeño comercio, la que más afecta a los medios locales (PRNoticias, 2011).

En segundo lugar, por una serie de innovaciones tecnológicas que afectan a todos los medios de comunicación (Scolari y otros, 2007), incluyendo la expansión del uso que hacen las empresas periodísticas de las tecnologías de la información, y especialmente, de Internet, modificando no solo las estructuras, servicios, operativa y necesidades de los medios, sino generando nuevos tipos de lectores, nuevos competidores, etc. Y en tercer lugar, por el consumo que están haciendo los usuarios de las noticias periodísticas, cada vez más a través de la red y cada vez menos a través del papel, lo cual ha cambiado incluso no solo la forma de consultar estos medios locales, sino también, en parte, a los usuarios que acceden a los mismos.

Por una parte, muchos de sus lectores, sus oyentes y sus espectadores acceden a los medios por vías distintas a la lectura de periódicos en papel, recogidos en las redes de transporte público; o a la escucha de las emisoras de radio durante los trayectos de ida y vuelta del trabajo; o a la visión de la televisión en momentos determinados del día. Ahora, cada vez más, los usuarios de la información disponen ya en sus teléfonos móviles de aplicaciones que les permiten leer, visualizar y escuchar información actualizada cada vez en periodos más cortos de tiempo (PRNoticias, 2010). 
Si bien parte de la prensa local ya era en algunos casos gratuita, y tenían experiencia en recuperar la inversión necesaria mediante publicidad, los modelos de negocio rentables en Internet aplicados a la prensa aún están por llegar. Todavía no se ha encontrado la forma de amortizar la inversión de material, de tiempo y de personal necesarias para soportar la actualización continua de información en la red, incluso aunque las tecnologías empleadas sean cada vez más baratas.

Por otra parte, estamos ante cambios generacionales que afectan a los temas que interesan a los usuarios, a los medios de comunicación que prefieren, a sus formas y hábitos de lectura e incluso a los procesos de personalización, todo lo cual exige la necesidad de cambios en la actividad relacionada con las noticias locales. Son cambios, propiciados por las nuevas tecnologías que están variando incluso el propio concepto de "interés local" (Ventura, 2009).

\section{LOS MEDIOS LOCALES DE COMUNICACIÓN EN LA COMUNIDAD DE MADRID}

A esta suma de factores, ha de añadirse una incertidumbre adicional que afecta a los medios locales de comunicación en la Comunidad de Madrid. El hecho de que cubran un área que incluye la capital de España, sede de gran parte de los medios de comunicación nacionales, provoca que muchos de los acontecimientos que suceden en Madrid sean considerados de alto interés por estos medios.

Ello puede deberse a que un hecho sucedido en Madrid tenga alguna influencia sobre el resto de España; o bien, por un latente etnocentrismo: lo que importa a quienes viven en Madrid es visto como lo que también interesa en el resto de España, incluso cuando les sea ajeno porque se trate de un asunto meramente local.

Además, las noticias locales que se producen en Madrid suceden cerca de la redacción principal de los medios nacionales. Y estos medios, especialmente en tiempos de crisis económica, están cerrando delegaciones y corresponsalías en otras partes de España o del mundo, con el fin de recortar costes. Las noticias que suceden en la capital, o en los alrededores, son pues más fáciles y baratas de cubrir, porque no hay que disponer de personal adicional: si es preciso, pueden enviarse personal y equipos de la redacción central, sin necesidad de grandes gastos.

Por otra parte, la prensa local de Madrid, tiene una especial particularidad temática. En otros territorios, con menos población y más lejanos físicamente a las principales fuentes de poder nacional (político, económico, cultural, etc.), los temas de interés de la prensa local no son solo de ámbito municipal, sino que pueden tener alcance provincial o incluso regional.

Pero, en el caso de Madrid, además de la competencia de los grandes medios, se acumula la existencia de una gran cantidad de población, ya sea en los distintos barrios de la capital, o bien, en otros municipios o zonas geográficas. Por ello, los temas de interés de la prensa local madrileña tienden a ser exclusivamente municipales 
o, en el caso de la capital, incluso relativos solo a barrios o distritos. El resultado es que muchos medios locales madrileños tienen una agenda de proximidad mucho más pequeña y atomizada que en el resto de comunidades autónomas.

Por lo tanto, muchos medios locales de Madrid sí que cubren aspectos no incluidos en los contenidos de los grandes medios (que dedican solo algunas páginas, o solo algunos minutos al día). Sin embargo, todos estos medios, locales o nacionales, sí que compiten por una misma audiencia, en algunos casos, saturada de información.

La suma de todos estos factores ha supuesto que los medios locales de prensa, cuando se ha tratado de entidades realmente periodísticas con intención de permanencia en la Comunidad de Madrid, se hayan agrupado en conjuntos de medios, con el fin de aprovechar la sinergia entre ellos. Fundamentalmente, estos grupos han sido, o bien cadenas de periódicos locales (El Buzón, El Distrito, Crónica de Madrid o Gaceta Local, por citar algunos); o bien, la suma de periódicos y de emisoras de radio, como, por citar otro ejemplo, El Iceberg, cuyo grupo, además de varios periódicos locales, incluye una emisora de radio, Globo FM. Algunos de estos grupos llegaron a disponer de cierta capacidad económica a comienzos del siglo XXI (como El Iceberg o El Telégrafo, entre otros), lo cual incluyó proyectos de puesta en marcha de canales de televisión locales, que requieren una mayor inversión económica. Sin embargo, la llegada de la crisis económica ha hecho que buena parte de ellos hayan tenido que posponer estos planes de expansión y, al contrario, hayan reducido el personal y los medios disponibles, y con ello, a veces, el número de medios periodísticos puestos en circulación.

La bonanza económica durante las décadas de los 90 y comienzos del siglo XXI, así como la existencia de una gran audiencia potencial, debido a la gran concentración de población en la comunidad, sobre todo, en la capital y en los municipios más cercanos a la misma, llevaron a la creación de medios de comunicación (sobre todo, periódicos) financiados por empresarios ajenos al sector de la prensa; y en particular, por fortunas generadas en el sector de la construcción, que pretendían generar opinión favorable a sus intereses de todo tipo.

En cuanto a las emisoras de radio y de televisión, cuando han existido, se ha tratado sobre todo, bien de emisoras municipales públicas, financiadas por el Ayuntamiento correspondiente, y sometidas en muchos casos a la voluntad de los responsables políticos de turno, que han dependido de los resultados electorales; o bien, de emisoras que formaban parte de cadenas nacionales, en las que la información local se ha limitado a algunos momentos puntuales durante el día.

\section{PRINCIPALES RESULTADOS DE LA ENCUESTA}

El primer resultado obtenido de la encuesta, que condiciona en buena medida el análisis de todas las cuestiones planteadas en esta investigación, es que, en los medios analizados, con la excepción de Telemadrid y Onda Madrid, más autonómicos que 
locales, no existe un solo centro de documentación como tal, y por tanto tampoco hay prácticamente personal dedicado expresamente a la realización de tareas de archivo y documentación sobre los contenidos, ya sean informativos o de entretenimiento, que contienen esos medios. En todos los medios se habla siempre del archivo, del fondo generado por ellos mismos y que les sirve no solo como memoria de lo publicado o emitido sino también como servicio para la consulta y para la reutilización de información.

A pesar de la inexistencia de un verdadero archivo o centro de documentación no se puede decir que los medios no sean conscientes del valor de la documentación, ya se trate de aquel material externo que requieren para la realización de sus contenidos, o bien, de sus propios contenidos, ni que dejen de utilizar documentación para sus contenidos casi de forma diaria, como puede extraerse de los resultados parciales que mostramos a continuación, organizados en los mismos epígrafes en los que hemos separado las preguntas realizadas.

\subsection{Los fondos documentales}

En 11 de los 12 medios consultados (con la excepción del ente autonómico formado por Telemadrid y Onda Madrid), "documentación" es el término que los periodistas locales asocian de forma incompleta, sin mencionarlo, al de "hemeroteca" o "fonoteca", que ellos denominan también como "archivo". Por una parte, casi todos los medios mantienen una hemeroteca o fonoteca de las publicaciones o programas difundidos, así como el archivo fotográfico del medio, en el caso de los periódicos. 9 de los 12 medios realizan ese archivo en soportes digitales.

Otro aspecto relevante sobre estos contenidos radica en su ubicación y, sobre todo, en su control y acceso. En 9 de los 12 medios consultados (75\%), es un miembro de la redacción con responsabilidad, sea un jefe de informativos, un redactor jefe o el propio director de la publicación, el encargado de custodiar físicamente ese archivo. Y, en 3 medios (33\%), es éste responsable el encargado de buscar y recuperar (es decir, de realizar las tareas documentales) para los demás redactores del medio.

Esta doble tarea, responsable de la redacción y encargado ocasional de la búsqueda de información textual, gráfica o sonora, suele derivarse de que al menos 6 de los medios citados $(50 \%)$ señalaron expresamente que tenían una alta rotación de personal: únicamente los cargos eran estables, mientras que los redactores de base eran becarios, o bien, periodistas recién licenciados en busca de mejores oportunidades de trabajo.

No obstante, para 7 de los medios citados $(58,3 \%)$, la custodia de estos fondos tiene más bien una mera función de archivo, es decir, de lugar donde se almacena físicamente esa información, como prueba de que ha sido publicada, por si fuera necesario recurrir a ella en un futuro a medio plazo, con fines históricos, culturales, etc.

Por ello, no todos los medios disponen de esos contenidos directamente en la redacción. Un $25 \%$ de los medios cuentan con un espacio fuera de la misma, aunque físicamente cercano, en el que se almacenan copias de los ejemplares publicados, más 
como un mero depósito físico de documentos, con un escaso tratamiento documental. Al menos otro $25 \%$ de los encuestados solo disponen en la sede de la redacción de la documentación más reciente, mientras que el resto es almacenada en un edificio lejano. Más aún: otro $25 \%$ de los medios han llegado a realizar expurgos masivos, eliminando de sus fondos contenidos publicados por falta de espacio o de presupuesto para su mantenimiento.

Los contenidos gráficos y sonoros, que suelen estar en soportes digitales, sí se mantienen en la redacción, aunque en casi todos los medios consultados, hay una única copia de cada documento. En el 33\% de los medios encuestados, el acceso a esos documentos está en un servidor externo. Ninguno de los medios locales consultados (dejando al margen al ente autonómico) no disponen de presupuesto para contratar personal informático. Un $25 \%$ de estos medios han contratado con otras empresas el servicio de publicación en la web y el servicio de almacenamiento y búsqueda de información.

\subsection{Cómo se usan los fondos documentales}

De las entrevistas realizadas, puede desprenderse que, en 7 de los 12 los medios consultados $(58,3 \%)$, la mayor parte de las consultas documentales de fondos propios corresponden a la búsqueda de fotografías. Las fotografías están digitalizadas, y disponibles en los ordenadores de la redacción, en la mayoría de los medios. Aún así, los redactores realizan también consultas en los fondos gráficos de otros medios y fuentes, en general, directamente accesibles a través de Internet, casi de forma diaria.

El archivo apenas se utiliza para rellenar los contenidos textuales, escritos o sonoros (33\%). En estos casos el uso principal del archivo es como fuente de resolución de dudas (nombres, cargos, fechas...). Por las características del periodismo local, los contenidos que se ofrecen proceden fundamentalmente de las administraciones públicas, las asociaciones vecinales, los partidos políticos u otras organizaciones (equipos de deportes, asociaciones de comercio, etc.).

En al menos 5 de los medios consultados $(41,6 \%)$, cuando se trata de acontecimientos que tienen un largo recorrido, los redactores sí realizan búsquedas retrospectivas en sus contenidos ya elaborados, e incluso los responsables informativos se preocupan por identificar con etiquetas las noticias sobre ese acontecimiento (básicamente, lugar del suceso, nombre del personaje y una etiqueta general describiendo el tema), para facilitar su recuperación.

En cualquier caso, casi todos los medios impresos guardan colecciones completas en papel, de sus contenidos; el 50\% de ellos, además, almacenan versiones digitalizadas de sus documentos más recientes (entre sus tres y sus seis últimos años), en formato PDF. En cuanto a los contenidos sonoros, las emisoras analizadas disponen de una fonoteca bien organizada, y en muchos casos, digitalizada, que emplean de forma diaria. Radio Villalba, además, registra y ofrece las grabaciones de los plenos del ayuntamiento, incluso de forma gratuita, para todo el que las demande. La emiso- 
ra trataba además, en 2011, de hacer accesibles esos contenidos directamente a través de la web.

En una gran parte de los encuestados (8 de los 12 medios consultados, el 66,6\%), los periodistas suelen guardar motu proprio la información producida ante la ausencia de centro de documentación, en formato digital. Solo por esa función preservadora ansían contar con un centro de documentación, ya que les evitaría esta tarea, pero no valoran su posible existencia por la gama de prestaciones que les pudiera aportar en su labor informativa. De hecho, en realidad, lo que esos medios pretenden es más un archivo que un centro de documentación en sí.

Por último, 8 de los medios consultados $(66,6 \%)$ recurren especialmente a los fondos documentales propios en determinados aniversarios, en los resúmenes de fin de año, y en otros acontecimientos históricos similares. Un $25 \%$ de estos medios ( 3 de los incluídos en la muestra) también han cedido gratuitamente información a otros medios (por ejemplo, a periódicos nacionales, cuando ha sucedido algún hecho de trascendencia general en sus municipios), o incluso colecciones completas a distintas instituciones. También se ha constatado en 6 medios (50\%) el intercambio de información entre los mismos y otros, ya sea como cortesía entre sí, o por la existencia de relaciones personales entre periodistas de distintos medios que trabajen incluso en la misma zona geográfica.

\subsection{La influencia de las tecnologías de la información}

Todos los redactores encuestados están habituados al uso de las tecnologías ya no tan nuevas. Internet es hoy para los periodistas una fuente básica y barata de acceso diario a todo tipo de contenidos, hasta el punto de que apenas ningún medio (salvo la radio y la televisión autonómicas) dispone de conexión a servicios o agencias de noticias. Internet parece haberlo sustituido todo.

Desde el punto de vista periodístico, Internet se ha convertido para los periodistas en un elemento de consulta imprescindible que permite una búsqueda rápida, directa y autónoma sobre una cantidad enorme de datos, hasta el punto de que, como anécdota, alguno de los periodistas encuestados para este estudio denominó específicamente a Internet como "un enorme centro de documentación mundial".

A pesar de todo, un 16,66\% de los periodistas encuestados ( 2 de los 12 medios incluídos en la encuesta) se muestran preocupados por la pérdida de calidad de la información e insisten en la imprescindible evaluación de la fiabilidad: uso de recursos informativos rigurosos; contraste de diferentes fuentes; ordenación y selección de los datos obtenidos, etc. Otro $16,66 \%$ de los entrevistados apuntan la tendencia hacia un futuro con nuevos medios digitales de acceso inmediato y sin contextualizar, en los cuales la documentación será más adecuada para los reportajes. Y otros dos representantes de los medios consultados insisten en la importancia de la seguridad informática de los sistemas y la garantía de reducir al máximo las posibles pérdidas de datos una vez que todo se hace y se conserva en formato digital. 


\subsection{La crisis económica y el futuro}

Prácticamente todos los encuestados (11 de los 12, un 91,6\%), señalan que la crisis económica ha afectado a la actividad diaria de sus medios: reduciendo plantillas, recortando proyectos económicos para su conversión en multimedia, e incluso planteándose la propia subsistencia del medio, acorde con la desaparición de numerosas cabeceras en todo el país. Dicha situación de carencia ha supuesto que estos medios de comunicación locales estén subsistiendo con muchas dificultades, prescindiendo de sus servicios de documentación, y están referenciando sus contenidos con las fuentes gratuitas de información accesibles en Internet.

Los periodistas ven como situación ideal la posibilidad de buscar ellos mismos sus propios contenidos: ya sea con un tratamiento documental previo, o bien, accediendo directamente a sus bases de datos y hemerotecas digitales, o bien, directamente a las fuentes de información accesibles a través de Internet.

Los propios medios también están considerando la opción de colocar sus contenidos y hemerotecas en la Red. Sobre todo, los periódicos ya lo están haciendo, tanto los impresos como los exclusivamente digitales; en el caso de los encuestados, al menos la mitad de los mismos así lo señalan. En ningún caso se han planteado cobrar por el acceso a sus contenidos, aunque tres de ellos $(33,3 \%)$ hacen hincapié en la necesidad de rentabilizar esta información, pero todavía no se ha planteado cómo hacerlo.

Estos contenidos que se vuelcan a Internet están siendo colocados sin ningún tratamiento documental. En el mejor de los casos (un 33\% de los medios analizados) se incorpora un buscador cuando se trata de contenidos textuales. En uno de los medios consultados, se han producido pérdidas por razones técnicas de contenidos destinados a la web. La desconfianza en la tecnología digital ha hecho que en dos de los medios encuestados $(16,6 \%)$ se continúe conservando la información en soporte papel.

Por otra parte, ante una pregunta expresa al respecto, ninguno de estos medios se ha planteado, en caso de mejora económica, la potenciación de sus contenidos documentales, ni la creación de un centro de documentación propio. Fundamentalmente, aquellos medios que plantean mejoras "documentales" (33,3\% de la muestra) lo hacen pensando más bien en mejoras telemáticas, capacidad de almacenamiento, velocidad de acceso, etc., entendiendo que, con ello, el impacto se traduciría en una mayor rapidez en la elaboración de las noticias más que de un aumento significativo de la calidad informativa. Además, los escasos 2 de los 12 medios que sí afirman querer contar con un documentalista señalan la necesidad de que éste fuera capaz de asimilar su trabajo a las características propias del trabajo periodístico, planteando ciertas reticencias ante ello.

\section{CONCLUSIONES}

La conclusión general del estudio indica que los medios periodísticos locales de la Comunidad de Madrid apenas realizan un tratamiento documental sobre los fondos de 
los que disponen. Este hecho no es solo fruto de que la crisis económica haya recortado los gastos de todas las redacciones, y que dicho recorte haya afectado, como es el caso de este mismo estudio en otras comunidades autónomas, como Cataluña y Galicia, a la existencia de esos centros de documentación. El estudio sugiere claramente que los medios de comunicación locales madrileños, a diferencia de los gallegos o catalanes, apenas han tenido centros de documentación como tales, tampoco en las épocas de bonanza económica.

4 de los medios consultados $(33,3 \%)$ han llegado a desarrollar bases de datos y a utilizar servidores para el volcado de contenidos en la web, pero sin excesivo control documental. De hecho, también en esos casos, los periodistas consultados siguen viendo a los documentalistas como personas encargadas más de la custodia y descripción prolija de los contenidos, esto es, de su archivo. Los documentalistas son también percibidos como una fuente de freno en el trabajo cotidiano de los periodistas, sometido habitualmente al estrés y a la presión de la actualidad informativa, más que como especialistas en el tratamiento de la información que podrían ofrecer un cierto valor añadido a las informaciones que se producen.

Una posible razón radica en que los periodistas locales tienen accesibles la mayor parte de las fuentes de información que consideran necesarias para su trabajo cotidiano y que, en muchos casos, consideran más que suficiente el volumen de información resultante. El desarrollo de Internet como fuente de información, en la mayor parte de los casos, gratuita hasta ahora, así como su propia conciencia como usuarios que buscan información de forma continua, ha hecho que no consideren necesaria la existencia en sus medios de profesionales dedicados a buscar y tratar la información de una forma diferente a la suya.

Por otra parte, si bien los periodistas locales son ávidos consumidores de información, también digital, las empresas periodísticas consultadas no han empleado todavía sus contenidos documentales de alguna forma que pueda ser rentabilizada más allá de la producción diaria o semanal de su producto.

A pesar de todo, sí existe actividad documental en prácticamente todos los medios: no ya solamente la búsqueda de fotografías, que aparece como el material más solicitado de forma retrospectiva, sino la elaboración de productos documentales $\mathrm{y}$, más aún, la consulta más o menos frecuente de material por parte de distintos redactores, de la misma manera que ocurre en otros tipos de medios de comunicación (MicóSanz, Masip-Masip, y García-Avilés 2009). Esta consulta resulta difícil de cuantificar, ya que ninguno de los medios analizados tiene control alguno sobre ello, aunque sí parece desprenderse de las respuestas reiteradas en los responsables de los distintos medios.

La descripción y recuperación de información está relacionada con el periodo de permanencia de los redactores en el medio. En el caso de aquellos medios que disponen de personal más veterano, ya sean redactores especializados en un municipio, por ejemplo, o redactores jefes que se mantienen en el medio, son ellos quienes mantienen recopilaciones de informaciones retrospectivas que oscilan entre los archivos más 
o menos clasificados y las bases de datos de contenidos. Son estos veteranos quienes no solo controlan esos contenidos, temiendo que otros no sean conscientes de su importancia, sino que también realizan a veces las búsquedas para otros redactores, cuando es preciso. En algún medio, paradójicamente, el volumen de búsquedas es tal que se produce una sobrecarga de los periodistas encargados de esa tarea.

Todos los medios son usuarios habituales de las tecnologías de la información, y por lo tanto, de los contenidos digitales, hasta el punto de que ya apenas almacenan otro tipo de contenidos. Más aún, en algunos casos, han realizado tareas de expurgo sistemático de sus contenidos en papel o en otros soportes analógicos, en el caso de las emisoras de radio; pero, en muchos casos, no han planteado al mismo tiempo estrategias mínimas de conservación de esos contenidos digitales, como la mera existencia de más de una copia de los mismos. Ello, unido al riesgo de obsolescencia técnica que tiene cualquier documento en soporte digital (Rothenberg, 1999), hace que los medios no sean conscientes de que, en pocos años, sus contenidos pueden ser todavía más difíciles de recuperar, como ya ha ocurrido en algún caso concreto.

Algunos medios que contratan fuera su servicio de publicación en la web disponen de copias digitales en los servidores de otras empresas pero en ningún caso se plantean estrategias de conservación o seguridad de los datos por si se diera el caso de que la empresa contratada cesara en el servicio por causas empresariales (cierre de la actividad), judiciales (embargo de los equipos de la empresa que presta el servicio) u otras causas.

Por otra parte, la actual situación de crisis económica, que afecta a la propia supervivencia de los medios, dificulta que puedan plantearse el valor añadido que podría darles el tratamiento documental de sus contenidos, dado que el mismo requeriría el empleo a corto plazo de recursos económicos de los que no disponen. No obstante, el principal inconveniente no es solo económico, sino cultural: los medios periodísticos locales madrileños analizados, salvo excepciones, tienen un cierto sesgo negativo hacia la entrada de profesionales de la documentación en sus redacciones, y no son en absoluto conscientes de cuál puede ser su valor en el seno de sus medios.

En el caso de los medios (Telemadrid y Onda Madrid) que forman parte del ente autonómico, la documentación, si bien está incluída en el servicio correspondiente y dispone de recursos notables (que incluía una plantilla de hasta 25 documentalistas, en la fecha de elaboración de la encuesta), también ofrecía posibilidades de mejora, incluyendo la escasa difusión de sus contenidos documentales en la web, o el uso de Internet como una fuente cada vez más frecuente de información, a pesar de su notable archivo audiovisual.

\section{AGRADECIMIENTOS}

La investigación en la que se ha basado este texto ha sido realizada en el marco del proyecto de investigación "Situación de los centros de documentación, su rentabilidad 
y uso, condiciones laborales y formación de los documentalistas en los medios de comunicación de ámbito provincial y comarcal en las comunidades autónomas de Madrid, Galicia y Cataluña” (DGYCYT Ref. SEJ2007-67677 / SOCI), dirigido por Eulàlia Fuentes i Pujol, catedrática de la Universitat Autònoma de Barcelona, y financiado por el Programa Nacional de Investigación Científica, Desarrollo e Innovación Tecnológica, del Ministerio de Ciencia y Tecnología, entre 2007 y 2010.

\section{REFERENCIAS BIBLIOGRÁFICAS}

ALFONSO-NOGUERÓN, Lola (2009). "De la videoteca al robot pasando por Tarsys. Nuevos sistemas de gestión multimedia en Radiotelevisión Valenciana". El Profesional de la Información 18, no. 3: 333-340. $<$ http://www.elprofesionaldelainformacion.com/contenidos/2009/mayo/12.pdf $>$ AQUESOLO VEGAS, José. 1996. "Situación de los servicios de Documentación de la prensa diaria de Andalucía". Cuadernos de Documentación Multimedia, $\mathrm{n}^{\circ} 5$. $<\mathrm{http}: / /$ www.ucm.es.strauss.uc3m.es:8080/info/multidoc/multidoc/revista/cuadern 5/aquesolo.htm>

ASOCIACIÓN PARA LA INVESTIGACIÓN DE MEDIOS DE COMUNICACIÓN (AIMC). Resumen general de resultados EGM. Octubre de 2010 a Mayo de 2011. Madrid: AIMC, 2011.

$<$ http://www.aimc.es//-Datos-EGM-Resumen-General-.html> [última consulta: $1 / 6 / 2012]$

CONESA, Alicia (2006). "Televisió de Catalunya. La evolución hacia el archivo digital". En FIAT/IFTA World Conference 2006 "Saving and sharing our audiovisual heritage. The age of television archives". Getafe (Madrid): FIAT-IFTA. $<\mathrm{http}$ ://archivesatrisk.org/restricted/madrid_2006/samedi_28/28oct_13_Conesa.pdf> COROMINAS, Maria (2009): Televisió local a Catalunya. Barcelona: Institut d'Estudis Catalans. Accesible en: $<$ http://www.portalcomunicacion.com/ocl/download/Corominas.pdf $>$

ESTRADA, NORA-GONZÁLEZ, Belén; PATALLO-FERNÁNDEZ, Cristina; PASTOR-BLANCO, Marcela (2009). "Servicio de documentación de la Televisión del Principado de Asturias (TPA)". El Profesional de la Información 18, no. 3: 326332.

$<$ http://www.elprofesionaldelainformacion.com/contenidos/2009/mayo/11.pdf $>$

DOMINGO, David (coord); ALBORCH, Federica; ARAÜNA, Núria (2009): La informació de proximitat als informatius televisius catalans. Barcelona, Tarragona: Observatori de la Comunicació Local (InCom-UAB), Departament d'Estudis de Comunicació (Universitat Rovira i Virgili). Accesible en: $<$ http://www.portalcomunicacion.com/ocl/download/Informe_Infolocal.pdf $>$ 
FRANGANILLO, Jorge (1999). "Els serveis de documentació a les televisions locals". Item: revista de biblioteconomia $i$ documentació, $\mathrm{n}^{\mathrm{o}} 25$, p. 64-81. $<\mathrm{http}$ ://www.cobdc.org/cgi-bin/intranet/itemdoc.pl?page=num25/jfranganillo.pdf $>$

FUENTES I PUJOL, Eulàlia. 1992. Els serveis de documentació dels diaris de Barcelona deu anys després. En 4es Jornades catalanes de documentació. Barcelona: SOCADI, p. 285-305.

FUNDACIÓ ESPAI CATALÀ DE CULTURA I COMUNICACIÓ (2009): $L$ 'impacte de les publicacions municipals en el sector privat de la premsa comarcal i local. Barcelona: Associació Catalana de la Premsa Comarcal Accesible en: $<$ http://www.premsacomarcaldigital.cat/ACPCDocs/impacte.pdf $>$ [última consulta: $1 / 10 / 2012]$

LÓPEZ GARCÍA, Guillermo (ed.) (2008): Comunicación local y nuevos formatos periodísticos en Internet: cibermedios, confidenciales y weblogs. València: Servei de Publicacions de la Universitat de València. Accesible en: $<\mathrm{http}: / /$ www.cibermediosvalencianos.es/ComunicacionLocal.pdf $>$ [última consulta: $1 / 10 / 2012]$

IZQUIERDO LABELLA, Luis (2010). Manual de periodismo local. Madrid: Fragua. LÓPEZ ELVIRA, Ma Jesús (2005). "O Servizo de Arquivo e Documentación da CRTVG". En Documentación e poder na sociedade da información: o papel dos arquivos e os observatorios, ed. Xosé López García y Rosa Aneiros Díaz, p. 297306. Santiago de Compostela: Consello da Cultura Galega. $<$ http://www.consellodacultura.org/mediateca/extras/documentacion_e_poder.pdf $>$ [última consulta: $1 / 10 / 2012$ ]

LÓPEZ-LITA, Rafael; FERNÁNDEZ BELTRÁN, Francisco y DURÁN MAÑÉS, Ángeles (2007). Congreso de Comunicación Local (ComLoc 2006): Nuevas tendencias en la prensa local. Castellón de la Plana: Universidad Jaume I, 2007.

MICÓ-SANZ, Josep-Lluís; MASIP-MASIP, Pere y GARCÍA-AVILÉS, José-Alberto (2009). "Periodistas que ejercen de documentalistas (¿y viceversa?). Nuevas relaciones entre la redacción y el archivo tras la digitalización de los medios". El Profesional de la Información, vol. 18, $\mathrm{n}^{\mathrm{o}}$ 3: p. 284-290. $<$ http://elprofesionaldelainformacion.metapress.com.strauss.uc3m.es:8080/media/e gpnwvuhmj14lcjtxxww/contributions/g/4/9/3/g493525010538277.pdf> [última consulta: $1 / 10 / 2012]$

PAUL, Nora (2009). "Elegía del centro de documentación de prensa". El profesional de la información, v. 18, $\mathrm{n}^{\circ} \quad 3, \quad$ pp. 249-253. $<$ http://www.elprofesionaldelainformacion.com/contenidos/2009/mayo/nora_paul en>

PEINADO MIGUEL, Fernando, FERNÁNDEZ SANDE, Manuel y RODRÍGUEZ BARBA, Dolores (2010). Análisis de la prensa gratuita en Madrid capital. Madrid: Ayuntamiento de Madrid-Asociación Española de Prensa Gratuita, 2010 $<$ http://eprints.ucm.es/11542/> [última consulta: 1/10/2012] 
PÉREZ FEIJOO, Paulino: La evolución de la televisión local en el sistema audiovisual hacia la desaparición de las emisoras independientes en Galicia. El caso Televigo, 1995-2007 [Tesis doctoral, 2008] $<$ http://www.portalcomunicacion.com/ocl/download/paulinoperez.pdf $>$ [última consulta: $1 / 10 / 2012]$

PRNOTICIAS. "La aplicación Iphone llegar a la prensa local madrileña". En: PRNoticias, $30 / 11 / 2010$.

$<$ http://www.prnoticias.com/index.php/component/content/article/1012destacados-otras-secciones/10062164-la-aplicacion-iphone-comienza-a-llegar-ala-prensa-local> [última consulta: 1/10/2012]

PRNOTICIAS. "Zenith Vigía confirma los temores: prevé un $0,3 \%$ de caída en 2011". PRNoticias, 30/05/11. Madrid. $<$ http://www.prnoticias.com/index.php/prmarketing/956/10067204> [última consulta: $1 / 10 / 2012]$

RAZQUÍN, Pedro. 1993. "Situación de los Centros de Documentación en los Medios de Comunicación de Madrid". Cuadernos de Documentación Multimedia, $\mathrm{n}^{\mathrm{o}}$ 2: $\mathrm{p}$. 71-78. <http://www.ucm.es/info/multidoc/multidoc/revista/num2/prazquin.html> [última consulta: $1 / 10 / 2012$ ]

ROS-MARTÍN, Marcos; RODERO-SUSIAC, Alfonso (2009). "El servicio de documentación de un medio impreso regional: evolución en el diario Levante-EMV". El Profesional de la Información 18, no. 3: 316-322. < $\mathrm{http}: / /$ www.elprofesionaldelainformacion.com/contenidos/2009/mayo/09.pdf $>$

ROTHENBERG, Jeff. Ensuring the Longevity of Digital Information. Santa Mónica (CA, Estados Unidos): RAND, $<$ http://www.clir.org/pubs/archives/ensuring.pdf> [última consulta: 1/10/2012]

SCOLARI, Carlos; JARQUE, José Manuel; PERALES, Cristina (2007): El canvi digital als mitjans de proximitat: les transformacions generades per la introducció de les tecnologies digitals en les redaccions de la premsa comarcal $i$ les ràdios $i$ televisions locals. Barcelona: Generalitat de Catalunya.

VENTURA, Borja. "Internet no se come a nadie. O por qué vale la pena apostar por la Red (también en el área local)". En: IV Jornadas Internacionales de periodismo. Elche: Universidad Miguel Hernández, 29 y 30 de abril de 2009. $<\mathrm{http}$ ://www.slideshare.net/azathyel/pmo-proximidad $>$ [última consulta: $1 / 10 / 2012]$ 


\section{ANEXO. CUESTIONARIO ENVIADO A LOS MEDIOS INCLUÍDOS EN EL ESTUDIO}

\section{CARACTERÍSTICAS DEL CENTRO DE DOCUMENTACIÓN}

¿Cuántas personas trabajan en el medio? ¿Cuántos periodistas colaboran con el medio? ¿Cuántos documentalistas trabajan en el medio?

¿Puede darnos información sobre categoría laboral y antigüedad del colectivo de periodistas que colabora en su medio? ¿Puede darnos información sobre categoría laboral y antigüedad del colectivo de documentalistas y empleados del servicio de documentación?

¿Cuál es la ubicación del servicio documentación dentro del medio? Si el servicio está alejado, ¿cree que una ubicación más cercana del centro de documentación mejoraría su uso por la redacción?

\section{USO DE LA INFORMACIÓN}

¿Cuáles son las principales necesidades informativas de los periodistas del medio? ¿Con que frecuencia formulan consultas al servicio de documentación?

¿De que recursos informativos disponen los periodistas para la realización de la información? ¿Cual es el horario de apertura del servicio?

¿Prevén aplicar mejoras para aumentar la calidad del servicio? ¿Qué propuestas realizadas por el servicio de documentación se podrían incorporar? ¿Y que propuestas realizadas por los periodistas?

¿Qué necesidades informativas cubre el servicio de documentación? ¿Con que frecuencia consultan los periodistas el servicio para la producción de información?

¿Qué carencias le han comunicado los periodistas en referencia al servicio de documentación? ¿Y qué puntos fuertes?

¿Ha generado el servicio de documentación productos de valor añadido? ¿Cuáles han sido? ¿Se han comercializado?

¿Ha generado el servicio de documentación algún producto específico para el medio? ¿Cuál? ¿Con que frecuencia? ¿A quien va dirigido? 
¿Ha generado el servicio de documentación algún producto específico servicios o empresas externas al medio? ¿Cuál? ¿Con que frecuencia?

\section{INFLUENCIA DEL USO DE LAS TECNOLOGÍAS DE LA INFORMACIÓN}

¿Cómo ha afectado el aumento y la inmediatez de la información al trabajo del servicio de documentación? ¿Ha modificado los hábitos de consumo de información de los periodistas? ¿Ha modificado la demanda de información? ¿y la relación con el servicio?

¿La extensión de las nuevas tecnologías de la información y la comunicación han afectado al consumo de información de los periodistas? ¿Ha variado el uso del servicio de documentación por parte de los compañeros del medio?

¿Este aumento de las NTIC ha variado la manera de trabajar de los empleados del servicio de documentación?

\section{INFLUENCIA DE LA CRISIS ECONÓMICA}

7. ¿Ha afectado la crisis económica en su medio al servicio de documentación? ¿Se ha mantenido el personal del servicio? ¿Ha aumentado? ¿O ha disminuido?

8. ¿Se ha mantenido el volumen económico de recursos de que dispone para la prestación de servicios? ¿Cómo prevén que afectaran estos cambios a la calidad del servicio? ¿Y de la información producida?

9. ¿Se ha notado la crisis de ventas y difusión en su medio? Si es así, ¿ha aumentado o ha disminuido el papel del centro de documentación en la elaboración de informaciones?

10. ¿Ha afectado la crisis económica en su medio al servicio de documentación? ¿Se ha mantenido el personal del servicio, ha aumentado, disminuído...?

11. ¿Se ha mantenido el volumen económico de recursos de que dispone para la prestación de servicios?

\section{EL FUTURO}

20. ¿Cómo prevé que será el futuro de los servicios de información en los medios de comunicación? ¿Qué papel considera que juegan los servicios de documentación de los medios de comunicación en el producto final que se ofrece al consumidor? ¿Prevé que cambiará en un futuro próximo? ¿En que sentido? 
21. ¿Qué impacto calculan sobre la calidad de la información producida de un aumento del $20 \%$ de los recursos destinados al servicio de documentación? 